\title{
Abstraktion zum Anfassen
}

\section{Zwanzig Jahre mathematische Realität mit GeoGebra}

\author{
Dirk Huylebrouck
}

\begin{abstract}
2021 feiert die frei zugängliche, kostenlos benutzbare dynamische Geometrie-Software GeoGebra ihr zwanzigjähriges Bestehen. Sechs Experten sprechen über ihre Lieblingszeichnung in GeoGebra.
\end{abstract}

Dynamische geometrische Systeme ermöglichen es, Punkte und Linien in mathematischen Zeichnungen auf dem Bildschirm zu bewegen, ohne die Zeichnungen ständig überarbeiten zu müssen. Durch die Kombination dieser Funktion mit ,Computer-Algebra-Systemen' ist eine benutzerfreundliche mathematische Software entstanden, was ein Traum vieler Mathematikschüler*innen und -lehrer ${ }^{\star}$ innen war. Im Jahr 2001 wurde dieser Traum Realität, als der Österreicher Markus Hohenwarter, ein Masterstudent für Mathematik und Informatik an der Universität Salzburg, die Software GeoGebra entwarf und dann sowohl an der Florida State als auch an der Florida Atlantic University weiterentwickelte. Die Software ist bis heute frei zugänglich.

GeoGebra hat sich im Internet schnell verbreitet, so dass eine große Open-Source-Community entstanden ist. Der Ungar Zsolt Lavicza gründete zusammen mit Hohenwarter das International GeoGebra Institute an der School of Education der Johannes Kepler Universität in Linz (Österreich). Ziel war es, Lehrer, Studenten, Softwareentwickler und Forscher bei der Verwendung, Entwicklung und Übersetzung von GeoGebra-Projekten zu unterstützen. Anfangs bestand Skepsis bei eingefleischten Mathematikern, die weiterhin auf ihr eigenes geliebtes
Computerprogramm schworen: Mathematica, entwickelt von Stephen Wolfram (Illinois, USA), das eine Reihe sehr leistungsfähiger mathematischer Formeln enthält, oder Maple von der Firma Waterloo Maple (Ontario, Kanada), die die Computeralgebra auf ein höheres Niveau gebracht hat. Für den Unterricht ist jedoch das auf der GeoGebraPlattform frei verfügbare Lehrmaterial entscheidend. Es gibt Workshops und Projekte zu GeoGebra, was es zu einer vielseitigen Ressource gemacht hat, die nicht zuletzt in vielen MINT-Fächern (Mathematik, Ingenieurwesen, Naturwissenschaften und Technologie) genutzt wird. Die GeoGebra-Community reicht jetzt vom Kindergarten bis in die Universitäten und hat derzeit mehr als 1 oo Millionen Nutzer.

Trotz mehr als zwei Millionen öffentlich verfügbarer Ressourcen entwickelt sich GeoGebra immer noch in neue Richtungen wie Augmented Reality, 3D-Druck, Online-Übungssysteme und virtuelle Lerneinheiten. GeoGebra ist in Hunderte von Lehrbüchern und OnlineLernumgebungen auf der ganzen Welt integriert. Vor kurzem hat auch die STEAM-Community (STEM + Art) begonnen, ihr Potenzial zur Schaffung mathematischer Kunst zu erkennen. Wird die Mathematik endlich an den Kunstschulen ankommen?

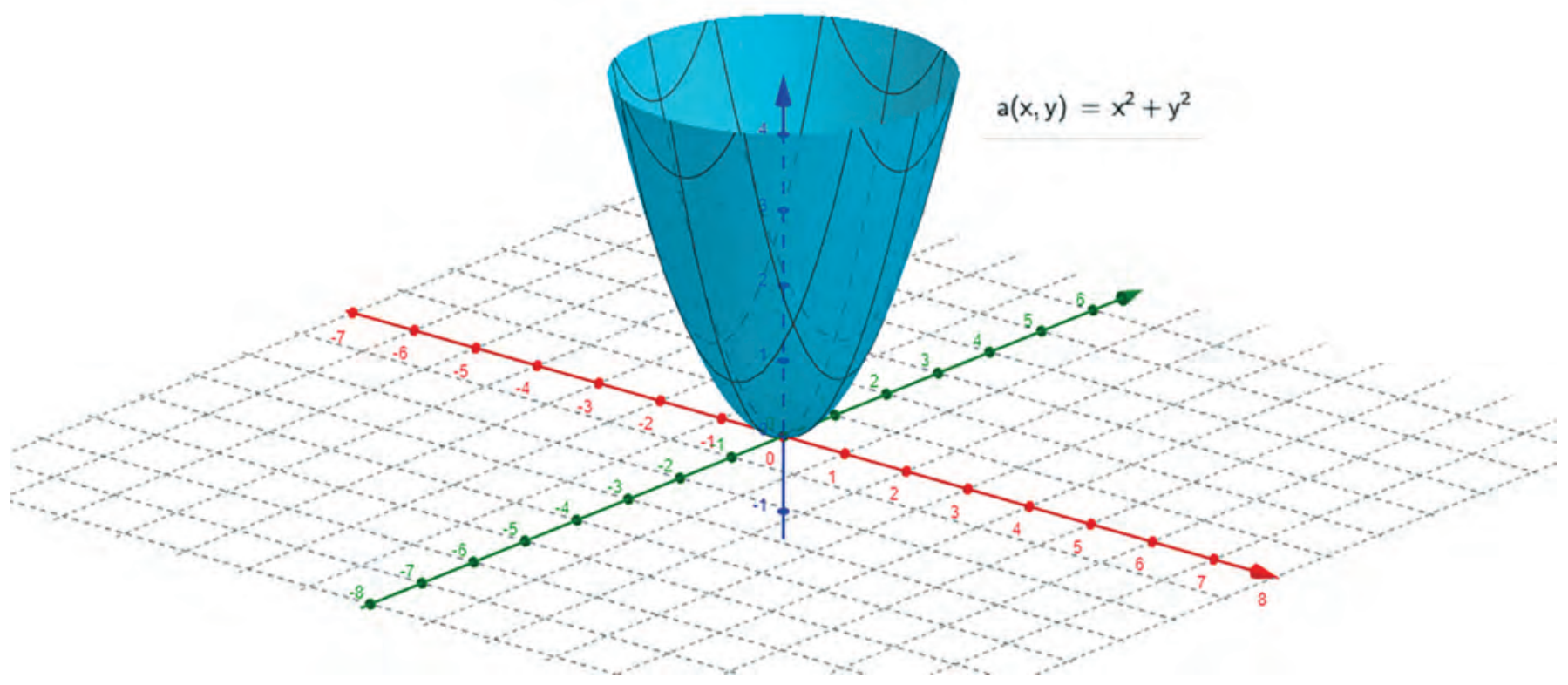




\section{Was sehen wir auf Ihrem Lieblingsbild in GeoGebra?}

Es ist ein Fraktal, das um 1942 vom niederländischen Mathematiklehrer Albert E. Bosman (1891-1961) erstellt wurde. Am Anfang beginnt man mit einem Quadrat als Stamm für den Baum und platziert dann zwei kleinere Quadrate in einem Winkel von $45^{\circ}$ darauf. Dieser Vorgang wird immer wieder wiederholt für die neuen Quadrate. Nach zehn Schritten, wie in dieser Zeichnung, ist das Ergebnis dem Querschnitt eines Brokkoli sehr ähnlich. Vielleicht hätte es der pythagoreische Brokkoli heißen sollen?

Warum illustriert dieses Bild (einen Teil der) Mathematik für Sie?

Fraktale erzeugen Neugierde. Darüber hinaus hat dieses Fraktal eine direkte Verbindung zum Satz von Pythagoras, dem geometrischen Satz, den fast jeder kennt.

\section{Was bedeutet dieses Bild für Sie persönlich?}

Dieses „GeoGebra-Applet“ zeigt deutlich, dass wir derzeit viel mehr Optionen haben, um Mathematikunterricht zu veranschaulichen als in der Vergangenheit. Sie können den Schieberegler (den Balken oben links) nach links oder rechts bewegen und so weniger oder mehr Brokkoli darstellen.

\section{Ben Haas: ${ }_{3}$ D-gedruckte Polygone}

Was sehen wir auf Ihrem Lieblingsbild in GeoGebra?

Mein Lieblingsbild zeigt verschiedene Polygone, die im GeoGebra 3D-Calculator mit Augmented Reality entworfen und dann mit dem 3 D-Drucker gedruckt wurden. Unsere Schüler rekonstruieren Muster in beiden Darstellungen. Ein Schüler kann eine Aufgabe für einen anderen Schüler auf sehr spielerische Weise erstellen. So verwenden wir sehr oft den GeoGebra 3D-Calculator zusammen mit Augmented Reality und 3D-Druck in der Sonderpädagogik im Kindergarten (3-6 Jahre) und der Grundschule (6-12).

Warum illustriert dieses Bild (einen Teil der) Mathematik für Sie?

In diesem Bild werden Symmetrie und Muster bearbeitet. Darüber hinaus stehen räumliche Orientierung, Polygone und deren Eigenschaften im Vordergrund.

\section{Was bedeutet dieses Bild für Sie persönlich?}

Das Bild zeigt, dass wir die reale und die digitale Welt leicht kombinieren können, um die Mathematik besser zu

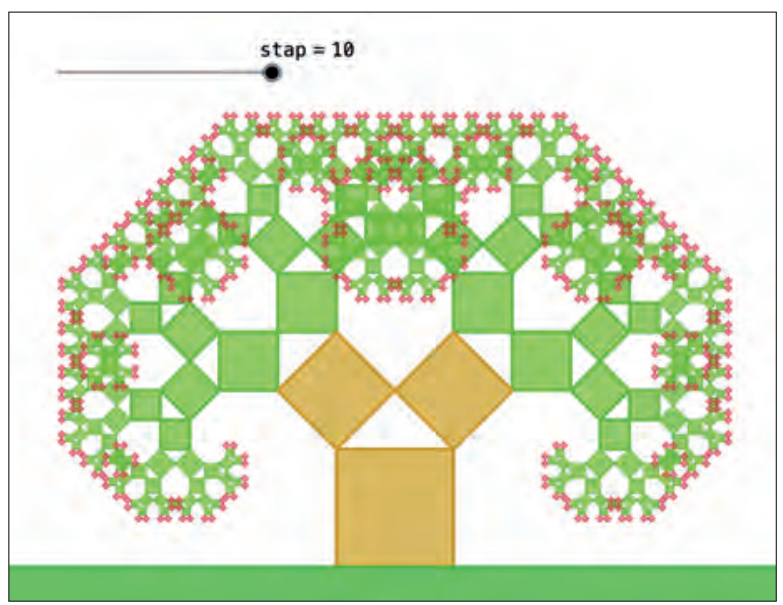

Wie blicken Sie auf die vielen Jahren zurück, in denen Sie GeoGebra verwendet haben?

Ich habe meine ersten Schritte in GeoGebra im Jahr 2006 gemacht und damals habe ich das Programm nur zum Erstellen von Zeichnungen verwendet. Später habe ich die dynamischen Funktionen von GeoGebra entdeckt, mit denen man zeigen kann, welche Änderungen ein Parameter verursacht. GeoGebra ist zu einem unverzichtbaren Bestandteil eines zeitgenössischen Mathematikunterrichts geworden!

Els Coussement ist Dozentin für Mathematik im „Bachelor in High School Education" an der Arteveldehogeschool Ghent, Belgien.

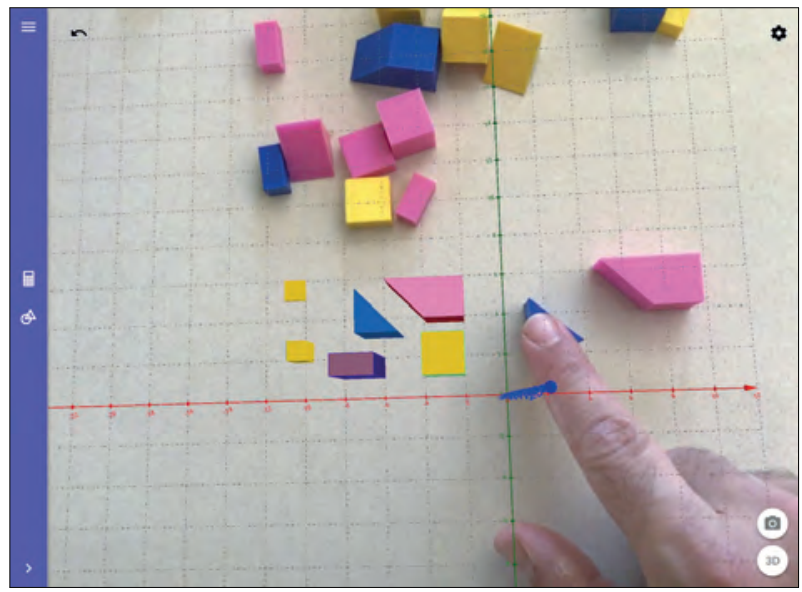

verstehen. Mit dem GeoGebra 3D-Calculator (mit Augmented Reality und 3 D-Druck) können die Schüler neue Fähigkeiten und ein tieferes Verständnis von Mathematik erwerben. Darüber hinaus zeigt mir meine persönliche Erfahrung, dass Schüler mit besonderen Bedürfnissen weit mehr lernen können, als oft erahnt wird. 
Wie blicken Sie auf die vielen Jahren zurück, in denen Sie GeoGebra verwendet haben?

Im Laufe der Zeit hat GeoGebra viele interessante Entwicklungen gemacht. Ich freue mich sehr, dass es immer mehr in Grundschulen und in der Sonderpädagogik eingesetzt werden kann. Es macht Spaß, damit als Lehrer zu arbeiten, und es gibt immer noch so viele weitere Möglich- keiten, die wir noch nicht kennen. Mit GeoGebra können wir mehr denn je ein besseres Verständnis der Mathematik schaffen.

Ben Haas ist Doktorant an der Linz School of Education, JKU, Lehrbeauftragter an der Universität Luxemburg, und Förderlehrer an der Millermoaler Schull, Luxemburg.

André Heck: Mathematisches CSI

Was sehen wir auf Ihrem Lieblingsbild in GeoGebra?

Dieses Foto, das von einigen meiner Schüler gemacht wurde, zeigt den Tatort eines inszenierten Verbrechens, betitelt ,eine Leiche im Flur'. Ein perspektivisches Quadrat, dessen tatsächliche Größe bekannt ist, wurde hinzugefügt. So kann beispielsweise der Abstand zwischen der Ferse des rechten Beins des Opfers und der Tür bestimmt werden, obwohl es nicht direkt mit dem Lineal gemessen werden kann. Das Erweitern und Verfeinern des perspektivischen Quadrats ermöglicht genaue Entfernungsmessungen auf dem Foto. Der geometrische Ansatz zeigt sich an den Linien, die die Fluchtpunkte und den Horizont definieren.

Warum illustriert dieses Bild (einen Teil der) Mathematik für Sie?

Das Bild zeigt eine Anwendung der Mathematik in der Forensik, die auf die in der Renaissance entwickelte Perspektivmathematik zurückgeht. Diese Mathematik ist in Computerprogrammen und Smartphones integriert, sodass man digitale Fotos bearbeiten und Messungen daran vornehmen kann. Jedoch bildet die projektive Geometrie die Grundlage für diese Methoden und Techniken.

\section{Was bedeutet dieses Bild für Sie persönlich?}

Ich habe den Artikel „Mathematical CSI“ in einer Zeitschrift für Mathematiklehrer*innen geschrieben, und anscheinend war dieser Artikel inspirierend. Mathematik wird spannend und macht Spaß, weil sie aktiv geübt und

\section{Zsolt Lavicza: Mathematische Augmented Reality}

\section{Was sehen wir auf Ihrem Lieblingsbild in GeoGebra?}

Dieses Bild wurde zusammen mit Ben Haas gemacht. Es zeigt den Bau eines kleinen Holzhauses mit Sandkasten in Augmented Reality. Mit dem GeoGebra 3D-Calculator können die verschiedenen geometrischen Körper der Objekte in der realen Welt identifiziert werden. Es wurde auf einem iPad ausgeführt und mit der Augmented Reality-Funktion verifiziert. Für eine korrekte Konstruk- durch ICT-Umgebungen wie GeoGebra unterstützt wird, wo dies möglich und notwendig ist.

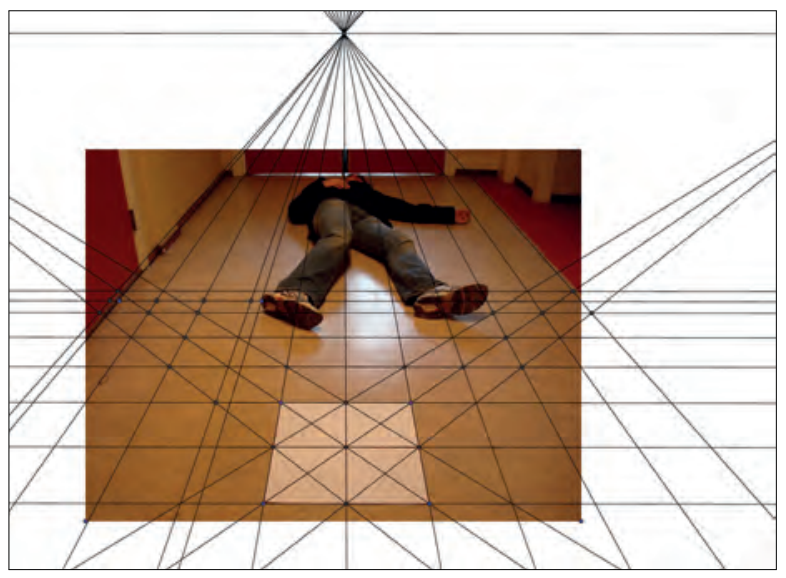

Wie blicken Sie auf die vielen Jahren zurück, in denen Sie GeoGebra verwendet haben?

Von Anfang an war ich von GeoGebra fasziniert, um Realität in den Mathematikunterricht zu bringen. Es war auch faszinierend mitzuerleben, wie immer wieder neue Entwicklungen und Möglichkeiten durch GeoGebra entstanden. Zum Beispiel untersuche ich derzeit die Einbettung des Systems in eine digitale Lernumgebung. In einem Satz: „Mit GeoGebra ist es nie langweilig."

André Heck ist Dozent für Mathematik und Forscher in der mathematischen Ausbildung am Korteweg-de Vries Institute for Mathematics, University of Amsterdam, Niederlande. tion müssen die Eigenschaften der Formen bekannt sein, die Liniensegmente müssen berechnet und die Strukturen moduliert werden.

Warum illustriert dieses Bild (einen Teil der) Mathematik für Sie?

Es zeigt die Anwendung der Theorie des ungarischen Mathematikers Zoltan Paul Dienes (1916-2014), der die 


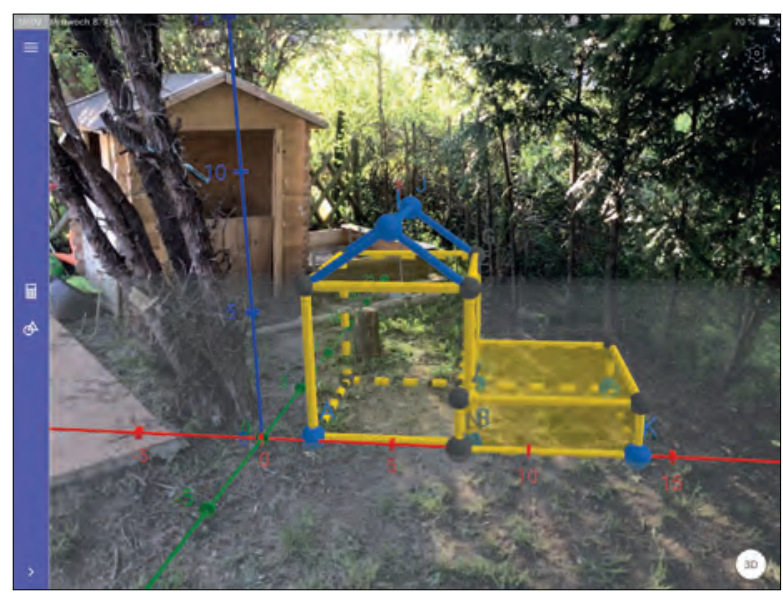

Verwendung manipulativer Prozesse betonte, die zur Abstraktion führen. Koordinaten, Liniensegmente, Formen und ihre Skalen werden zuerst in einer konkreten Umgebung bearbeitet, dann abstrakt manipuliert und kehren dann zur konkreten Situation zurück.

Was bedeutet dieses Bild für Sie persönlich?

Doru Păunescu: Abstraktion visualisieren

Was sehen wir auf Ihrem Lieblingsbild in GeoGebra?

Es repräsentiert einen komplexen Logarithmus, mit mehrwertiger Funktion. Für jede komplexe Zahl $z$ (ungleich Null) entspricht sie einer abzählbaren Anzahl unterschiedlicher Punkte, die durch

$$
\log [z]=\ln |z|+i(\arg (z)+2 k \pi), k \in \mathbb{Z})
$$

gegeben sind. Bei jeder vollständigen Drehung des ZPunkts um den Ursprung kehrt der Logarithmus zum Ausgangsstatus zurück, jedoch auf einer anderen Ebene.

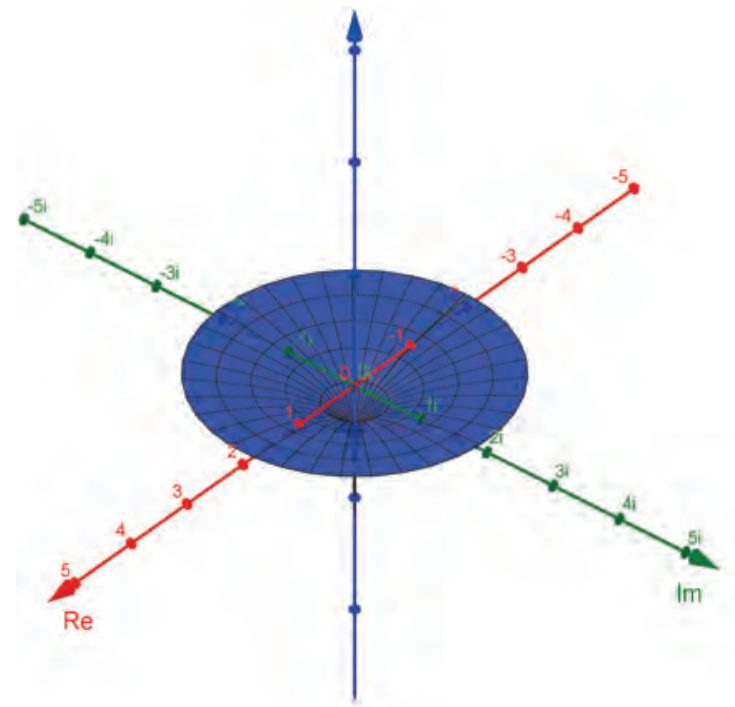

Das Bild zeigt, dass Mathematik nicht außerhalb des Lebens liegt, sondern mitten drin. Es ermöglicht uns, unsere Umgebung zu verändern und zu modulieren. Wir lernen, wie man mathematische Fähigkeiten und Kenntnisse auf die Umgebung, in der wir alle leben, anwendet. Wir werden aktive Gestalter in der Mathematik und bleiben nicht nur Bewunderer.

Wie blicken Sie auf die vielen Jahren zurück, in denen Sie GeoGebra verwendet haben?

Es war fantastisch, die Entwicklung von Mathematik (-Unterricht) in der ersten Reihe zu erleben. Viele Leute denken, dass sich Mathematik und ihre Lehre nie ändern werden, da es die gleichen Formeln seit undenklichen Zeiten gibt. Die Wirklichkeit ist weit davon entfernt, wie GeoGebra uns gezeigt hat. Die Zukunft ist vielversprechend: Augmented Reality und Modellierung werden auch in der Grundschule noch stärker integriert.

Zsolt Lavicza arbeitet an der University of Cambridge und der Linz School of Education, Linz, Österreich.

Der Ursprung ist ein singulärer Punkt für den komplexen Logarithmus. Links im Bild ist der Realteil des komplexen Logarithmus und rechts der imaginäre Teil.

Warum illustriert dieses Bild (einen Teil der) Mathematik für Sie?

Das Bild zeigt das bizarre Verhalten der Exponentialfunktion in der komplexen Ebene gegenüber der realen Exponentialfunktion. So ist die Exponentialfunktion im Komplexen periodisch, wohingegen sie im Reellen inver-

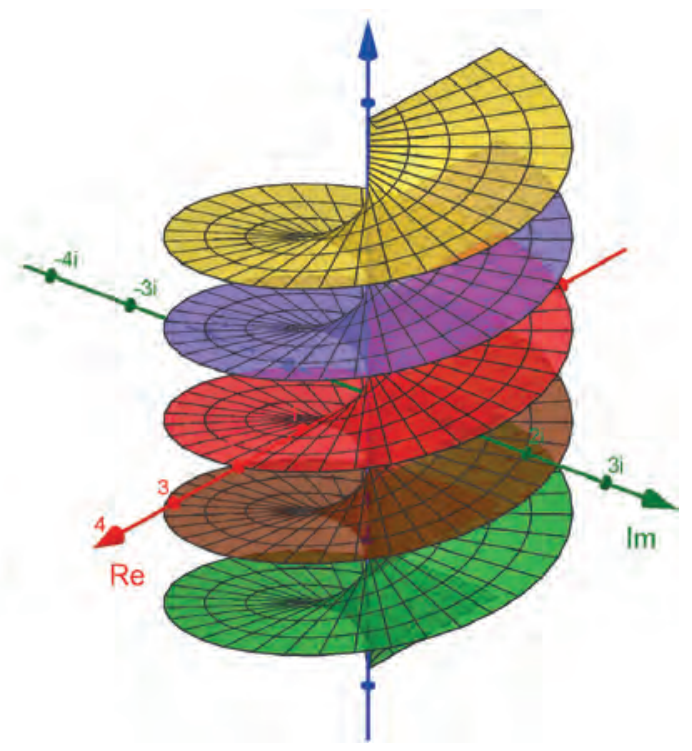


tierbar ist. Dies wurde mit GeoGebra in einem Buch von Juan Carlos Ponce-Campuzano illustriert.

\section{Was bedeutet dieses Bild für Sie persönlich?}

Ich persönlich mag GeoGebra vor allem aus zwei Gründen: (1) wegen der doppelten grafischen Ansicht, die es erlaubt, zwei Darstellungen gleichzeitig zu visualisieren, was das Studium verschiedener Arten von Transformationen erleichtert; (2) wegen der integrierten dynamischen Tools, mit denen Animationen erstellt sowie Grafiken oder Text gezeigt oder ausgeblendet werden können.

Simona Riva: Mathe schneiden

\section{Was sehen wir auf Ihrem Lieblingsbild in GeoGebra?}

Ich habe dieses Bild gewählt, obwohl ein Standbild GeoGebra nicht wirklich gerecht wird. Die Software zielt darauf, Mathematik in Bewegung zu zeigen.

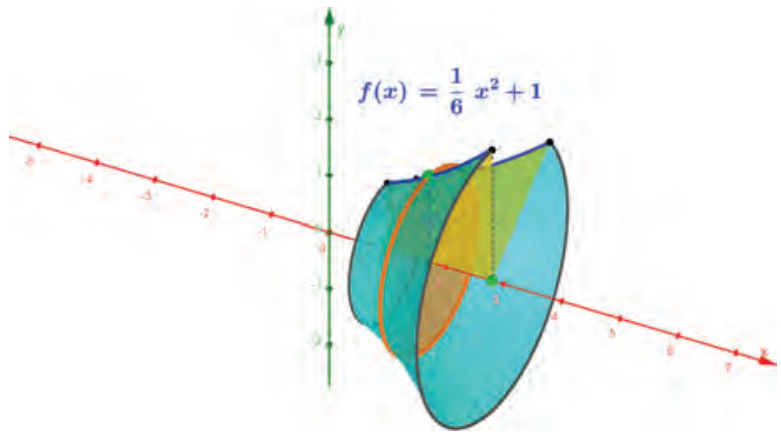

Warum illustriert dieses Bild (einen Teil der) Mathematik für Sie?

Das Bild zeigt, wie das Volumen eines Rotationskörpers durch Schneiden berechnet wird. Weil das ein dynamisches Konzept ist, ist es nicht etwas einfaches, was auf Papier dargestellt werden kann. Mit GeoGebra können Sie jedoch die Gleichung ändern, die den Bereich definiert, der gedreht werden soll, und in Echtzeit ein sich aktualisierendes Diagramm anzeigen, wodurch die Abschnitte visualisiert werden.
Wie blicken Sie auf die vielen Jahren zurück, in denen Sie GeoGebra verwendet haben?

Am GeoGebra-Institut der Politehnica-Universität von Timişoara versuche ich, die Unterrichtspraktiken zu verbessern und GeoGebra-Anwendungen auch für sehr abstrakte mathematische Begriffe zu entwickeln. GeoGebra ist ein wunderbares Werkzeug für Lehrer und Schüler, mit dem dynamische und interaktive Anwendungen auf vielen mathematischen Ebenen erstellt werden können.

Doru Păunescu ist Associated Professor am Department of Mathematics, Politehnica University of Timişoara, Rumänien.
Was bedeutet dieses Bild für Sie persönlich?

Ich habe dieses Bild vor einigen Jahren für meinen Sohn erstellt, der damals Calculus studierte. Bei einem Treffen meines Sohnes und seiner Freunde habe ich mitbekommen, wie sie meine interaktive Aktivität schätzten - mein Sohn prahlte damit, dass seine Mutter es erstellt hat. Ein solches Lob bekommt die Mutter eines Teenagers nur sehr selten zu hören!

\section{Wie blicken Sie auf die vielen Jahren zurück, in denen Sie} GeoGebra verwendet haben?

Es war Liebe auf den ersten Blick. Vor Jahren suchte ich nach einer Open-Source-Grafiksoftware und fand GeoGebra. Damals war es im Vergleich zu heute noch recht einfach, aber ich sah, dass es großes Potenzial hatte. Ich fragte Markus Hohenwarter, den Entwickler von GeoGebra, ob ich helfen könnte, die italienische Benutzeroberfläche zu verbessern, und wurde sofort in eine sehr unterstützende Community aufgenommen. GeoGebra hat die Art und Weise, wie ich Mathematik unterrichte verändert, weil ich mit einem Konzept interagieren und meine Aktivitäten mit Kollegen und Studenten teilen kann.

Simona Riva ist Autorin und GeoGebra Applets Designer für De Agostini Publishers, Italien. 